\title{
Knowledge Spaces
}

\author{
Marcos Baez, Fabio Casati, and Maurizio Marchese
}

Dipartimento di Ingegneria e Scienza dell'Informazione, University of Trento, Italy

\{baez, casati, marchese\} @disi.unitn. it

\begin{abstract}
This paper presents a set of models and an extensible social web platform (namely, Knowledge spaces) that supports novel and agile social scientific dissemination processes. Knowledge spaces is based on a model for scientific resources that allows the representation of scientific knowledge and meta-knowledge, of effective "viral" algorithms for helping scientists find the knowledge they need, and of interaction metaphors that facilitate its usage. The concept and a preliminary implementation of Knowledge spaces, in their various forms and designs, are being exploited in several different pilots.
\end{abstract}

Keywords: knowledge dissemination, social web, scientific publications.

Knowledge spaces (kspaces for short) are a metaphor, a set of models and processes, and a social web platform that help you capture, share and find scientific knowledge, in all of its forms.

The principle behind kspaces is to allow knowledge dissemination in the scientific community to occur in a way similar to the way we share knowledge with our colleagues in informal settings. The rationale behind this is that when we interact informally with a small team of colleagues dissemination is very effective. We are free to choose the best format for communicating our thoughts and results, we share both established results as well as latest ideas, we interact and carry on a conversation (synchronously or via email), we comment on other people's contributions and papers and observe relations among various contributions. Even when we remain in the domain of papers, we often find that we come to know interesting papers not by doing a web search or scan the proceedings, but because we "stumble upon" them, that is, we have colleagues pointing them to us via email or mentioning them in a conversation (along with their comments), and knowledge spreads virally.

Kspaces aim at providing the models, processes, metrics and tools to support this informal and social way of disseminating knowledge among the scientific community at large and via the Web, complementing the well-established method of papers published in conferences and journals after peer review. The goal is to use a webbased system to enable the capturing of these evolutionary bits of knowledge and data, however they may be expressed, as well as the capturing of ideas and opinions about knowledge, and leverage this information and meta-information to spread knowledge virally. Capturing opinions on knowledge is particularly important. The fact for example that somebody (and especially somebody we "trust") shares a paper tells us a lot on the value of this paper, much more than a citation can do. As readers, we relate them, in our mind, with prior knowledge. When listening to a talk we think that other work is relevant to the one being presented and often we jot it down in our 
own personal notes. In a world where information comes out from the web like from a hose, this knowledge about knowledge becomes essential to dissemination. Tagging, annotating and connecting the dots (linking resources in a way much more useful to science than citations) become almost as important as the dots themselves.

Kspaces support this not only by using web technologies as the basis for its implementation but by using web 1.0 and 2.0 concepts in the way scientific resources and their relationships are modeled and in the way knowledge sharing is supported. In essence, kspaces is characterized by a conceptual model and a repository for scientific resources (or for pointers to them if stored elsewhere). Resources are linked in arbitrary ways and relationships are typed and can be annotated. This is analogous to the Web, although it is oriented to linking scientific resources and to supporting (and then leveraging) relationship types and annotations. Indeed building this evolving web of annotated resources and leveraging it to find knowledge is a key goal of kspaces. The intuition is that having such web of connected knowledge can be as instrumental or even more instrumental (because it contains more metadata) to finding knowledge than the Web is to finding web pages. Today this web of resources is simply not there and this is part of what makes finding interesting scientific knowledge hard.

On top of this space of resources, kspaces define specific processes, permissions, and interaction modes people use to share knowledge. Kspaces manifest themselves in various forms, called designs, tailored at capturing different forms of scientific knowledge shared in different ways, from maintaining a library of related work, talks, datasets, etc, in an area - including our own, evolving work - to forming knowledge communities, writing and publishing (liquid) books, supporting the collection of the knowledge that emerges in the mind of attendees during a talk, and many others. It is through spaces with specific design that knowledge and meta-knowledge is collected and disseminated. The dissemination and search of knowledge over kspaces is then based on the "social interest", on the goals of a search (e.g., related work vs introductory material), and on the meta-knowledge (e.g., tags and annotations). Kspaces, although being richer and more flexible than many existing systems, is not the first and only platform that exploits some form of social meta-knowledge to support search. Mandeley, citeUlike, and Connotea, just to name a few, all have some elements of this. We believe that the key to a successful platform here lies in how such meta-knowledge can be collected and how it is used, and here lies a key contribution of kspaces.

Accessing kspaces. Kspaces have been developed in the context of the LiquidPub project (liquidpub.org). They are the results of several attempts and failures at arriving at a model for capturing knowledge, which we initially tackled by trying to impose a specific knowledge collection mechanism (that is, a single, specific KS app). More details, demos, and applications are available at open.kspaces.net

Acknolwedgements. We ack the great contributions to the ideas and the code from all the liquidpub team, with particular thanks to Alex Birukou and to all our kspaces developers including Delsi Ayala, Simone Dalcastagne, Nicola Dorigatti, Lyubov Kolosovska, Muhammad Imran, Michele Lunelli, Aliaksey Minyukovich, Daniil Mirilenko, Alejandro Mussi, Cristhian Parra, Laura Pop. This work has been supported by the LiquidPub project (FP7 FET-Open 213360.). 